\title{
COLOURED PETRI NET MODEL FOR REMOTE MONITORING OF CARDIOVASCULAR DYSFUNCTION *
}

\author{
CARLOS ROVETTO, ELIA CANO, KARINA OJO, MODALDO TUÑON \\ Department of Computer Science, Universidad Tecnológica de Panamá, Panamá.
}

HECTOR MONTES

Faculty of Electrical Engineering, Universidad Tecnológica de Panamá, Panama; Field and Service Robotics Group, Centre for Automation and Robotics (CSIC-UPM), Spain

\begin{abstract}
Cardiovascular dysfunction is the main cause of death in the world and several of these incidents have not been detected in time for diverse reasons. Consequently, some research groups, in ICT area, have been developing remote monitoring systems to detect the biosignals of cardiovascular dysfunction patients in the recent years. In this paper, a Coloured Petri Net (CPN) model for the remote healthcare process is presented. This CPN model uses the patient data formalization by means of set theory, providing a simplified perspective of the data transmission among all elements of the system. Using the CPN model, it will be investigated the behavior the system formed by the patients and their biosignals, located in different city zones (e.g., Panama), by means of simulation processes, in a first stage. With obtained simulation results, a remote monitoring system of bio-signals using non-invasive sensors will be developed.
\end{abstract}

Keywords: Coulored Petri Net; Cardiovascular dysfunction; Remote monitoring system; Vital signs; Non-invasive sensors.

\section{Introduction}

The telemedicine field is changing very fast in different directions with the aim of offer healthcare to different kind of patients remotely located. As technology advances at exponential levels, so does the widespread affordability and accessibility to basic telemedicine tools. Nowadays, it is an advantage, in terms of time and resources, monitoring in remotely way to patients with any disease, especially people who present cardiovascular diseases (CVD) [1].

According to WHO [2], CVDs are the number 1 cause of death globally. An estimated 17.7 million people died from CVDs in 2015, representing $31 \%$ of all global deaths. Of these deaths, an estimated 7.4 million were due to coronary heart disease and 6.7 million were due to stroke. Over three-quarters of CVD deaths take place in low- and middle-income countries. People with CVD need early

\footnotetext{
* This work is supported by the Universidad Tecnológica de Panamá.

H. Montes et al. (eds.), CLAWAR 2018: 21st International Conference on Climbing and Walking Robots and the Support Technologies for Mobile Machines,

Robotics Transforming the Future

(c) ELSEVIER
} 
detection and management using counseling and medicines, as appropriate. Considering the two last assertions, in a particular case, it indicates in Panama is necessary to develop a project related to the remote monitoring of CVDs, with the objective to reduce the risk of premature death caused for this disease.

CVD is also the most invested economic resources of the public and private sector worldwide, which in Latin America exceeded, in 2015, 30 billion dollars, according to the data of the WHO offered in the World Congress of Cardiology and Cardiovascular Health (WCC 2016) in Mexico [3]. Additionally, in studies presented to the WHO [3], Panama has the fourth position, with people that suffer any CVD in Latin America, turning into a priority the improvement and the inclusion of monitoring techniques for these patients.

On the other hand, systems modeling is essential in the management of complex and variable systems (as the remote monitoring of CVD) due to allows understanding the different process that can occur through previous simulations, before the implementation of the system. In this case, a system model using Coloured Petri Nets (CPN) can be appropriate of the proposed system in this work. Because it has a graphical representation for an explicit description of states and actions. Moreover, offering hierarchical descriptions, formal analysis, and simulations [4]. These strengths will allow a detailed analysis of remote healthcare process using the proposed CPN model.

A Coloured Petri Net (CPN) model for a system that analyses the cardiovascular dysfunction patients, which they will be remotely monitored, is presented in this paper. This CPN model uses the patient data formalization by means of set theory, providing a simplified perspective of the data transmission among all elements of the system [5] (signals type, signals variability, location, alarms, etc.). Using the CPN model, it will be investigated the behavior the system formed by the patients and their bio-signals, located in different city zones, by means of simulation processes.

This work will be developed in Panama and it is part of the remote monitoring system for heart failure patients project presented in [6]. The rest of this paper is organised as follow: in Section 2 a general description of the proposed system is presented. In the next section, a proposed model using CPN is described. Finally, conclusions and future works are given in Section 4.

\section{Description of the system}

The main vital signs that will be measured, for carrying out this work, are cardiac frequency, respiratory frequency, arterial pressure, temperature, pulse oximetry 
and ECG. All used sensors are non-invasive and their data will be acquired by means of a wireless system.

The conceptualization of the full system is related to a perception of vital signs of a large number of cardiovascular dysfunction patients, located in several areas, e.g., at the hospital, at the city, at the home, at the workplace, and in rural zones. Figure 1 shows the conceived remote monitoring concept, which is divided into three levels, which are: Patient-level, Control centre level, and Healthcare service level.

Patients with non-invasive sensors can be in any place as mentioned above, and the sensors joined to them are connected to a wireless data acquisition system, which it sends the data to any data collector station established for this function, e.g., PC, smartphones, tablets, etc. However, depending on patient localization area, of the characteristic of the place, of the patient numbers, etc, a robotic system can be considered, in order to contribute to re-send the recollected data, to the patient surveillance, as a mobile healthcare assistant, etc. This robotic system would be included within the patient-level. Some researchers have considered the robotic systems into the automatic healthcare functions [7, 8]. Therefore the robotic system for this work will be very useful for the accomplishment of several tasks.

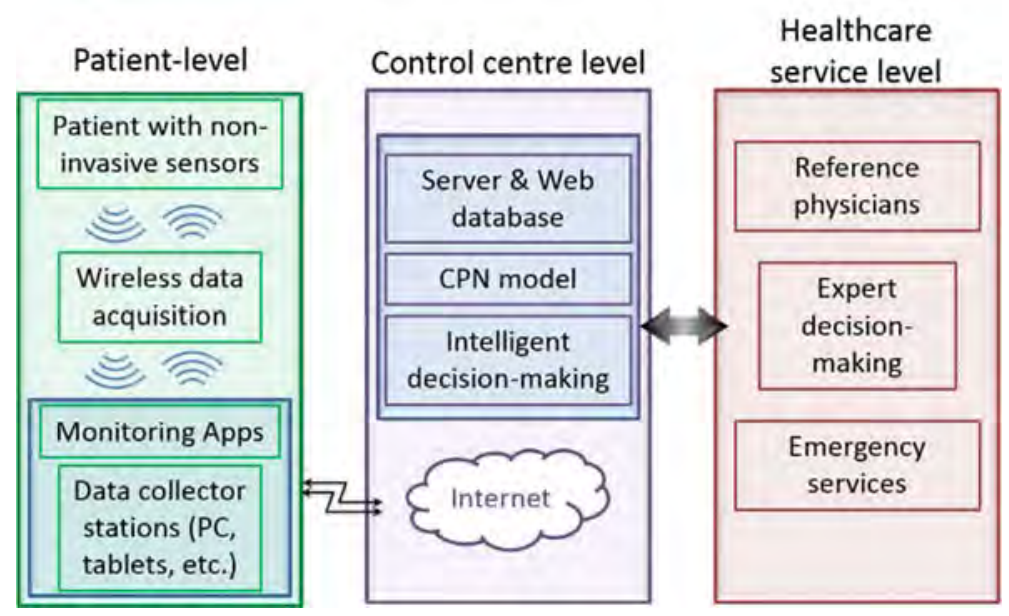

Figure 1. General overview remote monitoring system.

Control-centre level is compound by the network connection, specifically, the Internet, where is a server with a database, established previously with the first data received from the hospital centre. CPN model will be implemented into the server, which has been previously validated by means of several simulation 
processes. Intelligent decision-making will be carried out using the designed CPN model in order to provide to the next healthcare-service level relevant information in order to the experts take the final decisions.

\section{Proposed model using CPN}

The proposed CPN model shown in Figure 2 represents a basic structure and processes related to the Health Care Service. The circles represent states, the rectangles represent processes and the arcs indicate information flows.

This abstraction process catches basics elements using simplified way, due to limited space. Moreover, the control flow decisions are represented using basic data. However, the structure remains functional for other data. Uppercase variables represent colored tokens, and the lower case represents variables used during the data flows. Values enclosed in parentheses represent data entries provide by the sensors and established medical information.

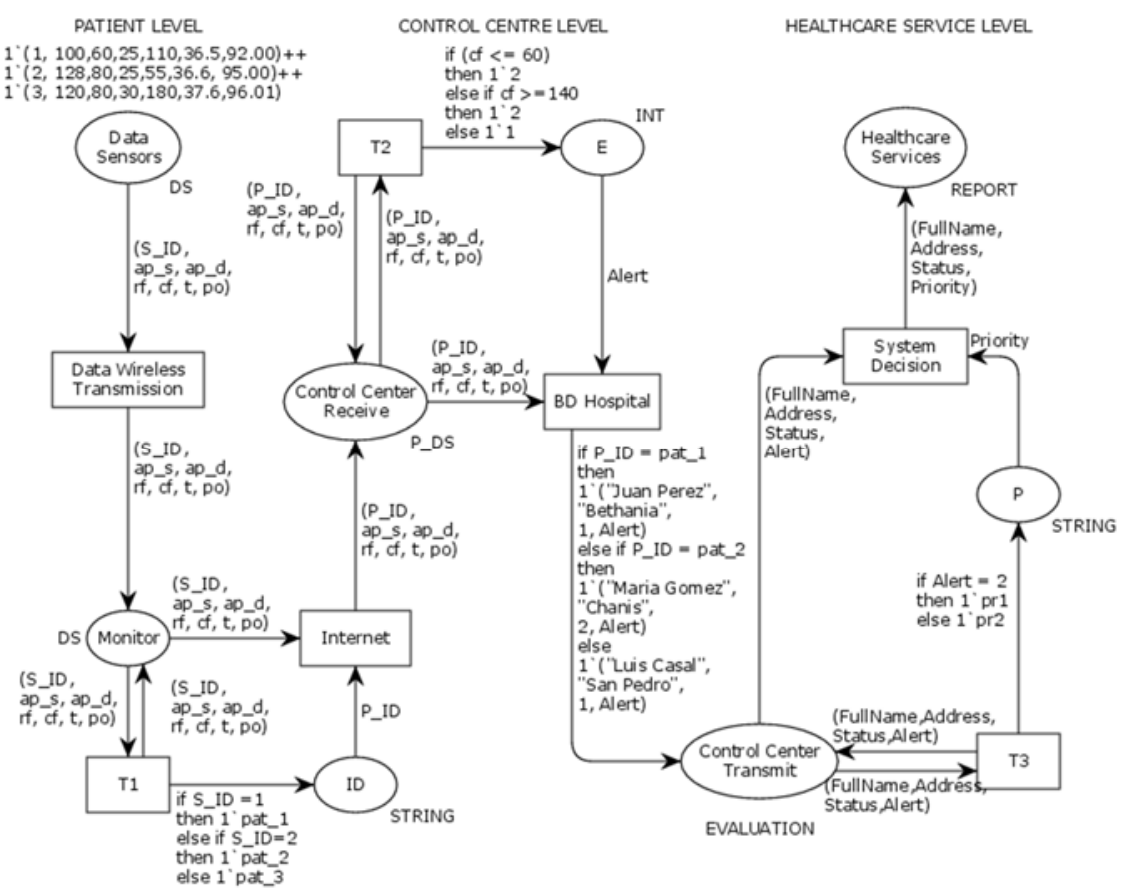

Figure 2. Coloured Petri Net Model.

Inputs parameters of the CPN net, shown in Figure 2, correspond to vital signs' measurements, i.e., systolic pressure (ap_s), diastolic pressure (ap_d), 
respiratory frequency (rf); cardiac frequency (cf); temperature (t) and oxygen saturation (po). These values are chosen in an aleatory way for simulation purposes with the CPN model.

The CPN model conserves the proposed stages or levels in Figure 1, whereby the process starts with data collection by non-invasive sensors (see "Data Sensors" in Figure 2). For simulation purposes, it is considered an identification number for each sensor (S_ID), which will be used to identify the patient (in the proposed CPN model in Fig. 2 has been considered three patients as an example). The monitor device that receives the data from the sensor carries out the patient identification process. The next step is to send this information by the Internet to the server of the Control Centre level (see "Internet" in Fig. 2).

In the server (see "Control Centre Receive" in Fig. 2), the received information is evaluated by taking into consideration the criteria charged and given previously by reference physicians, to ensure the highest accuracy in the decision-making. The CPN model shown in Figure 2 presents a specific example related to the evaluation for one of the vital signs of the patient, which is the cardiac frequency (cf). In this case, depending on the cf values received, the CPN model assigns an alert level, where the highest value represents a more critical situation of the patient.

Data's verification processes are represented in the CPN model using auxiliary places and transitions. For the patient identification (P_ID), the variables in the CPN model representing the following:

- $\quad$ T1 and ID verify the sensor's identification number.

- $\quad \mathrm{T} 2$ and E check the sensor's data and generate an alert (Alert) if necessary.

- $\quad$ T3 and P assign a priority (Priority) of being attended, based on clinical history and current patient status.

The final step consists of the intelligent decision-making by the proposed $\mathrm{CPN}$ model. The system generates a final report that is sent to Healthcare-Service level, which specifies the severity of the patients' conditions to physicians and experts in order to allow them taking action as fastest as possible. The proposed system could take other automatic decisions, based on intelligent decision-making such as (1) to send an ambulance toward the patient location, with independence if the patient is a different city where the system is installed; (2) to send a notification to a physician close to the patient with the collected data of him; (3) to send a notification to relatives if the patient is alone, etc.

CPN offers clear advantages for systems modeling, specifically for those systems where data transmission is critical for decision-making, as described above. This is because the CPN model has considerable strengths in visual 
representation and simulation processes, which allow understanding abstract processes, as data management in real time, in a simple way, where common datatypes can be used during simulations.

Using a CPN model foresees to raise the efficiency level for the remote monitoring of CVD within the system proposed in this work. Even more when it is considered to have a robotic system within the first level of the proposed system, which it will make the system more versatile because several automatic actions will be included in future works.

\section{Conclusions}

In this paper, a system for the remote monitoring of cardiovascular dysfunctions has been described, which will be implemented in Panama, due to the high rate of CVD in Panama and the Latin-American region. The main idea is to contribute to increasing the medical attention to a large number of patients and in that way reduce the risk of premature death. Therefore, the health specialist, using the proposed system, will be able to take the right decisions after the early diagnostics for the patients' disease.

This system includes a CPN model, which has not been used for this application type according to the literature reviewed. Several discrete events will be considered into the CPN model and will be simulated using several possible cases acquired by medical personnel. In this work, it has been presented a simple simulation using three patients, and it has been evaluated the cardiac frequency vital sign, as an example. It is expected the CPN model will provide good results when it has been applicated to several patients located in different places.

\section{Acknowledgments}

The authors thank the support of the Universidad Tecnológica de Panamá for the development of this project. Carlos Rovetto, Elia Cano and Hector Montes thank the National System of Researchers (SNI-SENACYT) of Panama by the support to their members. Hector Montes also acknowledges support from Centre for Automation and Robotics CSIC-UPM.

\section{References}

1. WHO, "A Health Telematics Policy," Report- WHO Group Consultation on Health Telematics - A Health Telematics Policy. pp. 1-39, 1998.

2. WHO, Cardiovascular diseases (CVDs). Media centre, WHO. (May 2017).

3. "El costo de las enfermedades cardíacas en América Latina.," Congr. Mund. Cardiol. y Salud Cardiovasc. (WCC 2016), 2016.

4. K. Jensen, A Brief Introduction to Coloured Petri Nets, (2005) 
5. K. Jensen and L.M. Kristensen, Coulored Petri Nets, Modelling and Validation of Concurrent System, (2009).

6. H. Montes, E. Cano, C. Rovetto, J. Saldaña, S. Vargas, Prop. of a rem. monit. sys. of vital sings heart failure patients, In Proc. CLAWAR 2018, (2018).

7. K. Boman, M. Olofsson, P. Berggren, P. Sengupta and J. Narula. JACC: Cardiovascular Imaging 7(8), (2014).

8. E. Wicklund, Telemedicine robots: Out of science fiction and into the mainstream. mHealth Intelligence, (October 2017). 